\title{
Migración haitiana en Santiago. Una aproximación multiescalar y temporal
}

Rosario Edwards. Pontificia Universidad Católica de Chile, Santiago, Chile. Margarita Greene. Pontificia Universidad Católica de Chile, Santiago, Chile.

RESUMEN | En el contexto del aumento de las migraciones hacia Chile provenientes de América Latina y el Caribe, que equivalen al 87,8\% del total de inmigrantes en el país al año 2017, el artículo se enfoca en la migración haitiana, una de las más singulares del último tiempo. Se aborda el habitar migrante en la ciudad de Santiago desde una aproximación multiescalar y temporal con métodos y escalas complementarias. Se describen los procesos de ocupación territorial de los principales colectivos a nivel de ciudad, se analizan las estrategias de inserción social y económica a nivel de barrio $y$, posteriormente, se identifican las configuraciones del grupo habitacional a escala doméstica. Analizar estas dinámicas migratorias a distintas escalas simultáneamente nos revela cómo las redes interpersonales organizan espacialmente las migraciones y modelan los espacios que las albergan en todos sus niveles.

PALABRAS CLAVE $\mid$ migración, redes, transformaciones socioterritoriales.

ABSTRACT | In the context of an increasing immigration to Chile from Latin America and the Caribe, which is equivalent to an $87,8 \%$ of the country immigrant population by 2017, this article focuses on the Haitian migration, one of the most significant of the last period. Migrant living is analyzed in Santiago city from a multiscalar and temporal approach with complementary methods and scales. We describe the processes of territorial occupation of the main groups at the city level, as well as the strategies of social and economic insertion at the neighborhood level. Afterwards we identify the configurations of the housing group at the domestic scale. Analyzing these migratory dynamics simultaneously and at different scales reveals how interpersonal networks spatially organize migrations and shape the spaces that host them at all levels.

KEYwORDs | migration, networks, socio-territorial transformations.

Recibido el 9 de junio de 2020, aprobado el 10 de octubre de 2020.

E-mails: R. Edwards,rmedwards@uc.cl|M. Greene,mgreenez@uc.cl 


\section{Introducción}

Desde el retorno de la democracia en 1990, Chile se ha consolidado como un país atrayente de flujos migratorios. Mientras el año 1992 se censaron 105.070 extranjeros residentes en el país, en 2017 esta cifra aumentó a 746.465 (Instituto Nacional de Estadísticas [INE], 2018). Esta realidad nacional es parte del fenómeno de la globalización, donde los flujos migratorios han alcanzado las cifras más altas de la historia. Del total de inmigrantes que recibe actualmente el país, cerca de $87,8 \%$ proviene de América Latina y el Caribe (INE, 2018), por lo que constituye predominantemente un flujo migratorio sur-sur. Dentro de este, adquiere especial interés el estudio de la migración haitiana, ya que presenta varias particularidades respecto a los otros colectivos latinoamericanos. Este proceso difiere tanto en los patrones idiomáticos, la composición racial y de género del flujo hacia Chile, como en las tendencias de localización en la ciudad y las configuraciones familiares.

La migración no es un fenómeno reciente; de hecho, las ciudades siempre se han construido a partir de ella, pero las migraciones contemporáneas presentan patrones distintos a las del pasado. La globalización ha modificado las migraciones de carácter definitivo, dando paso a proyectos migratorios a corto y mediano plazo que no siempre se materializan en un cambio definitivo de asentamiento (Stefoni, 2004). Muchas de ellas presentan movimientos temporales, circulares y multidireccionales que dan origen a nuevos estilos de vida y nuevos espacios transnacionales (Pries, 2005, en Organización Internacional para las Migraciones [orm], 2009).

En el Gran Santiago, donde se concentra el 65,3\% de los inmigrantes que recibe el país (INE, 2018), la intensificación de los flujos migratorios ha provocado una serie de fenómenos urbanos. Por ello, se llevó a cabo un estudio descriptivo desde una aproximación multiescalar y temporal en tres niveles -ciudad, barrio y vivienda-, buscando caracterizar el habitar migrante contemporáneo. Como plantea Stefoni (2004), las altas cifras y las nuevas dinámicas de las migraciones contemporáneas han obligado a buscar nuevos marcos teóricos para dar paso a una visión más compleja de estos movimientos. Así, y siguiendo la sugerencia de Çağlar y Schiller (2011), se utiliza la ciudad como punto de partida donde comparar y analizar la forma en que los migrantes y Santiago mutuamente constituyen y configuran un nuevo espacio urbano ligado a los procesos globales.

La concepción multiescalar hace referencia a los procesos socioespaciales que, organizados en torno a redes, se constituyen en múltiples escalas que abarcan el mundo entero (Çağlar \& Schiller, 2018). Si bien este enfoque expone que los migrantes sustentan localmente la reconstitución de procesos globales, el artículo se enfoca en las relaciones socioespaciales que se constituyen en los distintos niveles del Gran Santiago. Por su parte, la incorporación de la variable temporal en este análisis permite visualizar la construcción de estos espacios bajo la noción de proceso, cuestionando la visión estática de la ciudad.

Si bien en los últimos años se ha producido abundante literatura acerca del habitar migrante, pocas veces se intenta entender un colectivo en distintas escalas simultáneamente. Este enfoque busca aportar evidencia sobre la interrelación entre los distintos espacios de la migración, dando cuenta de cómo se ven modelados por 
las mismas relaciones sociales, estructuradas en torno a redes. A nivel de ciudad, las redes de connacionales organizan territorialmente las migraciones, agrupándose cada nacionalidad en determinadas zonas o comunas. A nivel de barrio, las estrategias de inserción social y económica específicas a su cultura de origen han establecido comercios y servicios con fuerte expresión en el espacio público e incorporado nuevas prácticas en espacios colectivos. Por último, a nivel de vivienda predomina la asociación de grupos habitacionales en viviendas colectivas, trascendiendo muchas veces los vínculos familiares y ligándose por redes adscritas a la misma comunidad de origen.

El enfoque propuesto permite avanzar en la comprensión de los procesos socioespaciales de adaptación y conflicto entre culturas diversas, que necesariamente conviven y se disputan el territorio urbano en todos sus niveles. Avanzar en su comprensión sentará las bases para el desarrollo de una estrategia urbana que ofrezca una solución al habitar migrante y aporte a la integración de esta creciente población en nuestro país.

El presente artículo consta de cinco secciones. Después de este acápite introductorio, en el segundo se presenta la metodología con que se llevó a cabo la investigación, y el tercero caracteriza las migraciones contemporáneas y el habitar migrante en las ciudades de recepción. En la cuarta sección se describen las tres escalas desde las cuales se analiza el fenómeno migratorio en Santiago. Finalmente, en la quinta se presentan las conclusiones y alcances del estudio.

\section{Metodología}

En este artículo se estudia el fenómeno migratorio abordando las escalas de la ciudad, barrio y vivienda con métodos complementarios. En la Figura 1 se describen los instrumentos, fuentes de información y método analítico, dando cuenta de la multiescalaridad y temporalidad del análisis.

En primer lugar, para entender los flujos migratorios en el Gran Santiago, se revisaron los registros de visa otorgados por el Departamento de Extranjería y Migración (DEM) entre los años 2005 y 2019, donde se consigna el ańo, nacionalidad y comuna de residencia de los migrantes. El análisis involucró la comparación de los flujos de llegada migratoria de las siete nacionalidades predominantes en el país -peruana, colombiana, venezolana, boliviana, argentina, haitiana y ecuatoriana- y un análisis de la primera localización de los cuatro colectivos más numerosos. La distribución de cada colectividad fue analizada y representada planimétricamente a través del tiempo (Figura 4).

En segundo lugar, se llevó a cabo una serie de entrevistas exploratorias a académicos expertos en el tema, funcionarios municipales y miembros de ong. De acuerdo con el análisis de localización en la ciudad y las entrevistas, se optó por trabajar en la comuna de Estación Central, de la Región Metropolitana de Santiago, y concentrar el análisis en el colectivo haitiano. La comuna se seleccionó debido a la alta presencia de migrantes, pero también por el hecho de ser la primera comuna de la zona central y pericentral de Santiago en obtener el "Sello Migrante". Esta distinción, realizada por el Departamento de Extranjería y Migración, identifica a los 
municipios que trabajan por la inclusión de la población migrante. Entre sus labores destaca la realizada por la oficina denominada "Estación Intercultural", dedicada especialmente a activar las redes de apoyo para integrar a las familias migrantes que residen en la comuna.

\section{FIGURA I | Estructura metodológica}

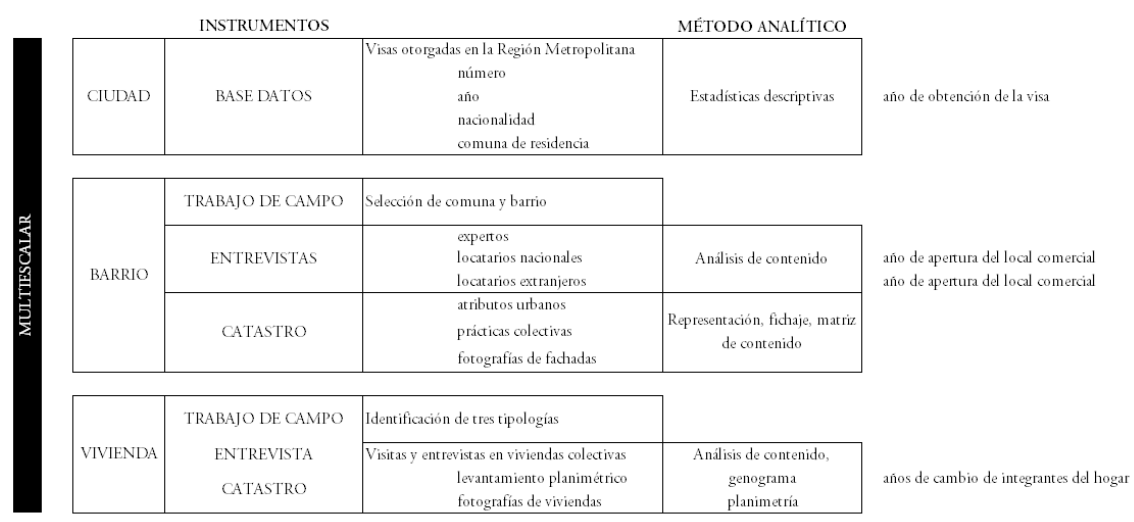

FUENTE: ELABORACIÓN PROPIA

Para la selección del barrio, se recurrió a las fichas de Protección Social de la comuna de Estación Central, las que señalan que los migrantes se concentran principalmente en 10 de los 59 barrios de la comuna. Se seleccionó como caso de estudio el barrio que presenta la mayor concentración de migrantes, que hemos denominado "Barrio v" a fin de mantener su anonimato. Se hizo un levantamiento sistemático de los principales atributos urbanos de dicho lugar (vialidad, morfología edilicia, vegetación, uso de suelos, red de transporte y espacios públicos), con especial énfasis en el catastro de los locales comerciales del barrio, dado que ha sido una de las manifestaciones espaciales más relevantes de la llegada de población migrante. Se consignó su año de establecimiento en el lugar, tipo de comercio o servicio y nacionalidad del locatario. Para visualizar el surgimiento y ubicación de los locales comerciales del barrio, se representó planimétricamente su distribución, clasificándolos según su origen en tres categorías -locales chilenos, locales haitianos y otras nacionalidades-, y se marcó en una línea de tiempo el año de su apertura (Figura 5).

Parte importante del método involucró la identificación y análisis de espacios colectivos utilizando el marco conceptual de Streetscapes (https://streetscapeterritories.org/). Esta perspectiva desafía la dicotomía tradicional entre propiedad pública y privada o formal e informal, y propone clasificar los espacios según como son cohabitados por diferentes actores que se relacionan entre sí y con el propio espacio. Así se incorpora al análisis de los elementos urbanos tradicionales, las lógicas emergentes que ellos encierran -como sus transformaciones físicas, usos temporales, 
apropiaciones y conflictos-, cuyos resultados materiales y simbólicos tienen la capacidad de resignificarlos (Chiappini et al., 2016).

El método consistió en anotar sobre los mapas de atributos urbanos, los usos, apropiaciones temporales, espacios simbólicos, actividades observadas en visitas sucesivas (idealmente hasta saturar la información que se encuentra en terreno) o mencionadas en entrevistas. El análisis posterior permitió identificar tres tipos de espacios colectivos que se clasificaron, según cómo se habitan, en espacios compartidos, cotidianos y emergentes (Urbina, 2016), y que fueron clave para la descripción analítica de la escala del barrio. Entre ellos se identificaron 112 negocios o almacenes, 89 de los cuales accedieron a responder una breve encuesta. También es importante señalar que este trabajo de campo se acompañó con imágenes fotográficas, que posteriormente se clasificaron, enriquecieron y dieron forma a las categorías de espacios.

Por último, dentro del mismo barrio y a través de las entrevistas y las visitas de campo, se identificaron tres tipologías de viviendas colectivas migrantes, clasificadas según su origen: galpones industriales, viviendas antiguas y vivienda social. Se realizó un levantamiento de una vivienda de cada tipología, identificando las fechas de las intervenciones y la cantidad de hogares, para reconstruir el proceso de ocupación de las viviendas. Además, para entender cómo los migrantes están habitando al interior de estas viviendas, se entrevistó a 18 personas de origen haitiano, revelando sus trayectorias residenciales y la composición de sus grupos habitacionales. Para ello, se contó con la traducción de un joven de origen haitiano que vive desde 2017 en el país.

\section{El fenómeno de las migraciones}

La globalización no solo ha elevado las migraciones a las cifras más altas alcanzadas en la historia en el país y en el mundo en general, sino que también ha transformado los modelos de asentamientos definitivos o colonizaciones, generando nuevas dinámicas migratorias de carácter temporal (Stefoni, 2004). Si bien las definiciones y los efectos de estas nuevas dinámicas aún están en una fase de análisis exploratoria, ya existe literatura que las caracteriza. Bovenkerk (1974) distingue distintos tipos de dinámicas según la flexibilidad de los movimientos y sus temporalidades, entre las cuales destaca la migración de retorno, la migración circular y la migración de tránsito. La migración de retorno ocurre cuando una persona vuelve a su lugar de origen, luego de haber emigrado por primera vez; cuando el movimiento entre dos países incluye más de un retorno, se denomina migración circular; y cuando la persona se mueve a un segundo destino, se trata de migración de tránsito.

También es importante señalar que la literatura actual declara que las migraciones contemporáneas se desarrollan transnacionalmente, en el sentido de que establecen relaciones sociales que cruzan fronteras geográficas, culturales y políticas (Schiller et al., 1992); y, más aún, que se caracterizan por su multiescalaridad. Con esto se refieren a que se constituyen entre múltiples escalas, desde lo local a lo global, manteniéndose interrelacionadas a través de redes sociales (Çağlar \& Schiller, 2018). De esta manera, las autoras no abordan a los migrantes como comunidades 
étnicas agregadas, sino como actores dentro y fuera del espacio en cuestión (Çağlar \& Schiller, 2011).

Es así como las relaciones sociales organizadas en torno a redes que se desarrollan interescalarmente modelan los espacios de la migración en todos sus niveles. De hecho, al decir de Brenner (2011):

ninguno de los espacios de migración -urbano, regional, nacional, transnacionalpuede verse como marco preexistente o estático de la vida social". No pueden entenderse como meros escenarios dentro de los cuales se posicionan o encierran las relaciones sociales. Más bien, todos estos espacios se ven producidos y coproducidos a través de las mismas relaciones sociales. (p. 37)

Las redes, como soporte de flujos sociales y culturales, no solo tienen una dimensión funcional, en la medida en que ayudan a acceder a servicios mediados por alguien de la red, como lo es el caso de la vivienda o el trabajo, sino que además estructuran la vida social en su conjunto (Imilan et al., 2014). En este sentido, Marcos y Mera (2018) exponen enfoques que han atribuido a las redes sociales y los lazos nacionales un rol predominante en el asentamiento en las ciudades de recepción, causando muchas veces concentraciones en determinados sectores urbanos. De esta manera, la segregación socioespacial en la ciudad no se reduce a un fenómeno socioeconómico y residencial, sino que la distribución de los migrantes en la ciudad y sus interacciones se articulan, además, por las complejas y cambiantes intersecciones de clase, género, raza, generación y residencia (Caggiano \& Segura, 2014).

Segura y Abde (2014) señalan que en la medida en que cada colectivo étnico utiliza su concentración en barrios como forma de protección, ayuda mutua y afirmación de su especificidad, se refuerza el patrón de segregación espacial. De esta manera, se establece una sensación de seguridad entre los migrantes dada por la apropiación de lugar y el conocimiento de las redes que en él se entretejen (Stefoni, 2015).

\section{Las tres escalas de la migración en Santiago}

\section{Tendencias de localización en la ciudad}

Las migraciones siempre han presentado patrones de localización relacionados con la preferencia por ciertas zonas en lugar de otras, pero dichos patrones han ido cambiando a lo largo del tiempo de acuerdo con la escala y estructura urbana de la ciudad. Históricamente, estas preferencias han privilegiado lugares con altos grados de conectividad que permiten el acceso a oportunidades laborales; con la existencia de un mercado de viviendas de arriendo de bajo costo, que se sitúa entre las más bajos de la jerarquía residencial; y, por último, y tal vez el factor más determinante, con la presencia de una red de connacionales, que se transforma en la primera red de inserción al país (Segura \& Abde, 2014). Esta red de lazos interpersonales de parentesco, amistad o pertenencia a la misma comunidad de origen, conecta a los migrantes, los migrantes anteriores y los no migrantes en áreas de origen y destino (Massey et al., 1993), sirviendo de medio de información para oportunidades, vivienda y empleo, y disminuyendo así los riesgos de la migración. Es más, las redes en el contexto migratorio muchas veces son causa de nuevos proyectos migratorios 
(Imilan et al., 2014), en la medida en que cada migrante incentiva a más connacionales a migrar.

Para visualizar la ocupación territorial de las migraciones en la ciudad de Santiago durante los últimos años, se analizaron las comunas de residencia declaradas por los migrantes al momento de tramitar sus visas (temporaria, sujeta a contrato y de estudiante) en el DEM entre los años 2005 y 2019, lo que representa los flujos temporales o bien la primera etapa de una inmigración de largo plazo. Se seleccionaron los siete colectivos migrantes predominantes en el país, todos provenientes de países de América Latina y el Caribe: Perú, Colombia, Venezuela, Bolivia, Argentina, Haití y Ecuador. En estos 15 ańos se entregaron 1.083.673 visas a ciudadanos de estas siete nacionalidades: la mayor parte a peruanos y venezolanos $(38,4 \%$ y $24,6 \%$, respectivamente), seguidos por colombianos y haitianos (13,9\% y $12,1 \%)$, y finalmente a ecuatorianos, bolivianos y argentinos (cada una con menos del 4\%). Por lo tanto, de estos siete colectivos, cuatro tienen una presencia mayoritaria, representando cerca del $90 \%$ del total de las visas analizadas (Figura 2).

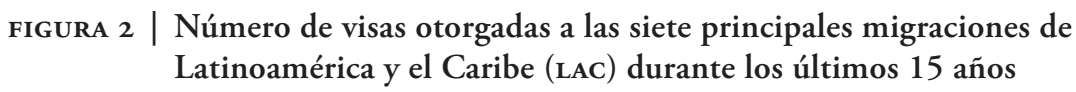

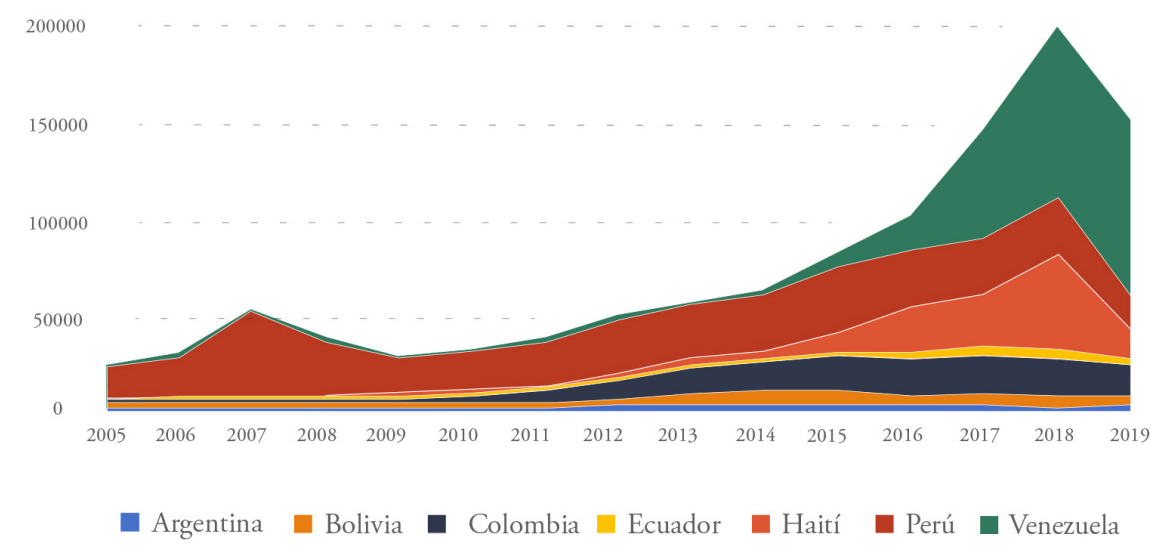

FUENTE: ELABORACIÓN PROPIA CON BASE EN DATOS DEL DEPARTAMENTO DE EXTRANJERÍA Y MIGRACIÓN (DEM)

$\mathrm{Al}$ analizar la residencia de estos cuatro colectivos al momento de recibir sus visas, se observa que el patrón de localización también diverge entre nacionalidades. Para este análisis se caracterizó la Región Metropolitana en cinco grupos: una zona periurbana, que incluye las 18 comunas periurbanas no conurbadas y las zonas rurales de las comunas que constituyen el Gran Santiago, y luego se dividieron las zonas urbanas de las 34 comunas del Gran Santiago en 9 comunas centrales o pericentrales, 6 comunas de alta renta, 15 comunas periféricas y 4 comunas con centros históricos conurbadas en el Gran Santiago (Figura 3a). El análisis de las visas entregadas entre los ańos 2017 y 2019 muestra que los colectivos de Perú y Venezuela siguen el patrón descrito por muchos autores: los migrantes privilegian el centro y 
el pericentro, buscando acceso a servicios y disponibilidad de alojamientos a bajo costo (alrededor del 60\% en estos dos casos). El caso de Colombia es bastante distinto, ya que, contra el argumento de la búsqueda de centralidad, la mitad se ubica en comunas periurbanas, no conurbadas con el Área Metropolitana de Santiago (AMS). Y el caso de Haití es aún más particular, ya que presenta una muy baja concentración en la comuna de Santiago (solo 8\%) y prácticamente se divide en zonas bastante equivalente: $30 \%$ en el centro y pericentro, $30 \%$ en la periferia y $40 \%$ en las comunas más alejadas del centro de la ciudad (20\% en comunas históricas conurbadas y 20\% en comunas periurbanas no conurbadas) (Figura 3b). Quizás el factor común de estas cuatro zonas donde se distribuye el colectivo haitiano, es que en todas ellas hay disponibilidad de vivienda a bajo costo.

\section{FIgURA 3 | Distribución de cuatro colectivos en la Región Metropolitana de Santiago}
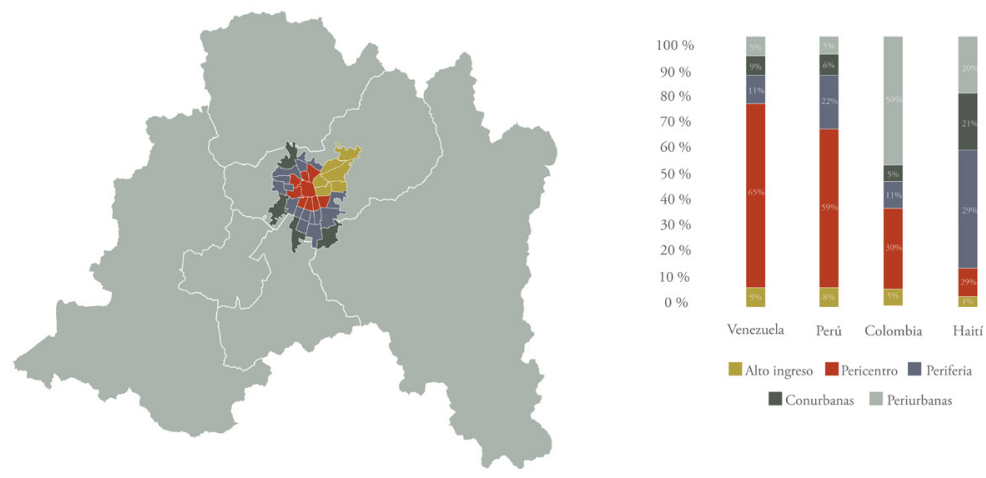

a) Región Metropolitana, distinguiéndose la zona periurbana y las zonas urbanas del Gran Santiago, categorizadas como de alto ingreso, pericentro,

b) Ubicación porcentual de los cuatro principales colectivos migrantes en la Región Metropolitana periferia, conurbanas y periurbanas.

según zonas anteriormente descritas.

FUENTE: ELABORACIÓN PROPIA CON BASE EN DATOS DEL DEPARTAMENTO DE EXTRANJERÍA Y MIGRACIÓN (DEM)

Posteriormente, se representaron las visas entregadas en el AMs por año y según comuna de residencia declarada al momento de realizar la solicitud. Cada visa fue graficada con un punto, y se distribuyeron homogéneamente en la superficie de cada comuna, reflejando, por ende, la cantidad y densidad comunal de visas otorgadas (Figura 4). La imagen muestra los años 2015 y 2018 para cada colectivo, dejando ver las tendencias residenciales al interior del Gran Santiago.

La Figura 4 muestra que todas las nacionalidades, menos la haitiana, presentan en mayor o menor medida un patrón concéntrico, alcanzando el mayor número de visas en Santiago centro. Si se analizan las cifras del ańo 2018, las comunidades venezolana, colombiana y peruana concentran en la comuna de Santiago el 41\%, 29\% y $24 \%$ del total de visas entregadas a su colectivo en el Gran Santiago, respectivamente. 
La haitiana, en cambio, muestra solo un $8 \%$ en esta comuna. De hecho, la mayor concentración de visas de este colectivo se sitúa en Quilicura, una comuna periférica conurbada al Gran Santiago. Esta comenzó a recibir los primeros flujos de migración haitiana el año 2006 (con 13 visas entregadas), liderando desde aquel entonces la mayor concentración de este colectivo, que alcanzó a 5.929 visas el año 2018.

\section{Figura 4 | Plano comunal del Gran Santiago, donde se señalan las visas entregadas los ańos 2015 y 2018 por colectivo migrante}
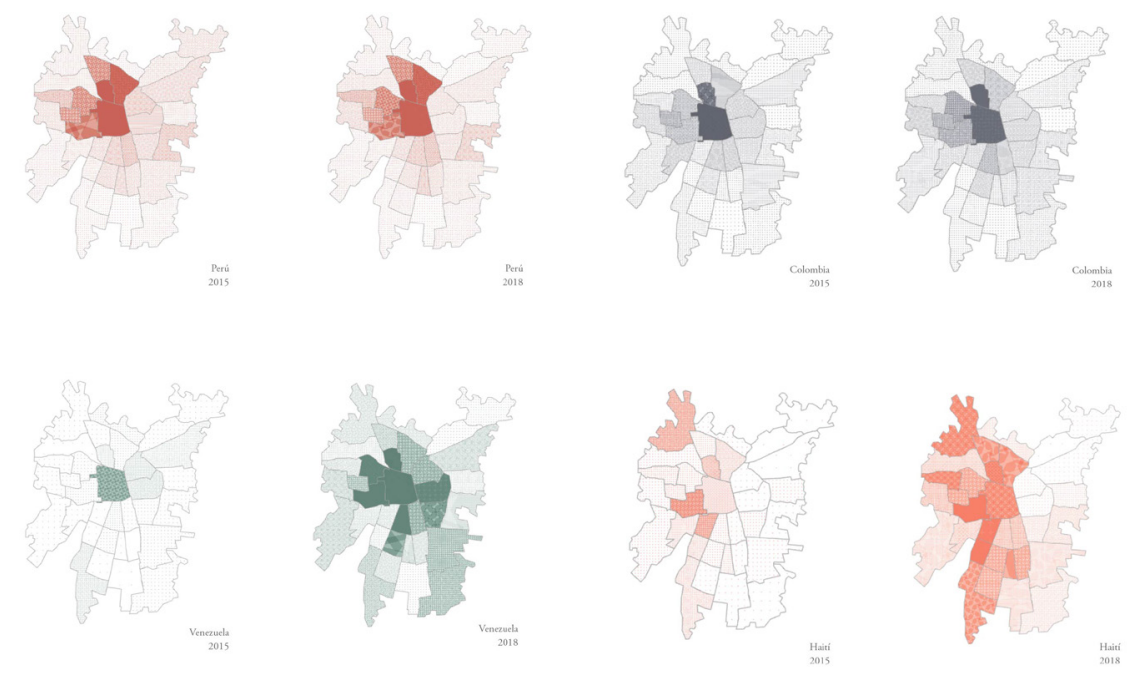

FUENTE: ELABORACIÓN PROPIA CON BASE EN DATOS DEL DEPARTAMENTO DE EXTRANJERÍA Y MIGRACIÓN (DEM)

Así, el estudio de la ocupación territorial a nivel de la ciudad refleja que tres de los principales colectivos migrantes corroboran lo expresado en la literatura sobre el tema. En ellos, la tendencia de localización se focaliza en lugares que combinan altos grados de conectividad, un mercado de arriendo de habitaciones de bajo costo y la presencia de redes de connacionales. Sin embargo, la comunidad haitiana presenta una dispersión en prácticamente toda la ciudad, con excepción del cono de alta renta, evidenciando sus mayores concentraciones en zonas distantes entre sí. Una posible explicación de este patrón de distribución es que en el colectivo haitiano los atributos de centralidad y conectividad se subordinan al acceso a vivienda de bajo costo, aunque, como se verá a escala de barrio, manteniendo cercanía con una comunidad de connacionales.

\section{Paisajes de la migración en el barrio}

En la comuna de Estación Central, los migrantes se concentran en 10 de los 59 barrios que la conforman (Vargas et al., 2015). Se seleccionó como caso de estudio el Barrio v, caracterizado por ser el de mayor concentración de migrantes, donde 
se estima que un $41 \%$ de su población es de origen extranjero, versus el promedio de 7\% a nivel metropolitano (INE, 2018). Esta elevada concentración se entiende por su buena accesibilidad a servicios, fuentes de trabajo y presencia de mercado de arriendo de bajo costo, lo que atrajo a los primeros migrantes. Luego el fenómeno se retroalimentó e incrementó producto del rol de atracción que ejercen las redes interpersonales y como una estrategia de apoyo y protección contra la discriminación.

El Barrio v seleccionado presenta un cierto grado de deterioro físico, con inmuebles abandonados, industrias menores y bodegas entre las viviendas y espacios públicos, en mal estado. En la medida en que ha ido recibiendo población migrante se ha intensificado la ocupación de los espacios públicos, afectado en gran parte por el alto nivel de hacinamiento de las viviendas migrantes. De esta manera, no solo se ha diversificado la población del barrio, sino que también se han introducido en el paisaje urbano prácticas cotidianas que no eran habituales. En este sentido, se entiende el fenómeno como un proceso de hibridación cultural, que da vida a un paisaje que sintetiza lo material con lo simbólico (Imilan, 2014).

La lectura de este paisaje urbano a la luz de Solá Morales (1992), muestra que hay espacios que "no son ni públicos ni privados, sino ambas cosas a la vez". Asimismo, se desafía la dicotomía entre propiedad pública y privada, formal e informal; y de acuerdo al marco teórico de Streetscapes, se reconocieron tres tipos de espacios colectivos en el Barrio v, clasificados según como se habita en espacios compartidos, espacios emergentes y espacios cotidianos.

El primer caso, los espacios compartidos, comprende los negocios y almacenes, porque a pesar de su carácter de uso de suelo privado, se constituyen como lugares de sociabilización del barrio. Para representar cómo la población migrante ha aportado a la proliferación de este tipo de espacios, se catastraron todos los comercios y servicios del barrio, identificándose un total de 112 comercios (Figura 5a). Del total de estos, 89 locatarios accedieron a responder la encuesta, identificándose 39 comercios impulsados por migrantes (44\%), de los cuales 23 eran haitianos y 16 de otras nacionalidades ( 11 peruanos, 3 dominicanos, 1 colombiano y 1 ecuatoriano). Considerando que la tasa de omisión por parte de la población migrante es alta, debido a irregularidad migratoria y, en el caso del colectivo haitiano, a la barrera idiomática, se estima que cerca de la mitad de los comercios del barrio son operados por migrantes, predominando los de origen haitiano. Es en este tipo de espacio donde los migrantes han alcanzado uno de sus mayores grados de visibilidad, dada la cantidad de negocios que han impulsado como estrategia de inserción económica.

En la Figura 5b se presenta el año de establecimiento del local comercial, identificándose los nacionales, los haitianos y los otros migrantes. Los datos señalan que el primer comercio migrante data del año 2001; que desde el 2015 hay un aumento explosivo de locatarios nuevos; y que desde el 2017 en adelante la mayoría de los locatarios nuevos son migrantes. El proceso del barrio hace eco a las palabras de Mehrotra (2016) cuando se refiere a fenómenos contemporáneos que moldean el espacio construido a velocidades extremadamente rápidas. 
Figura 5 | Catastro y línea de tiempo de locales comerciales en el Barrio v

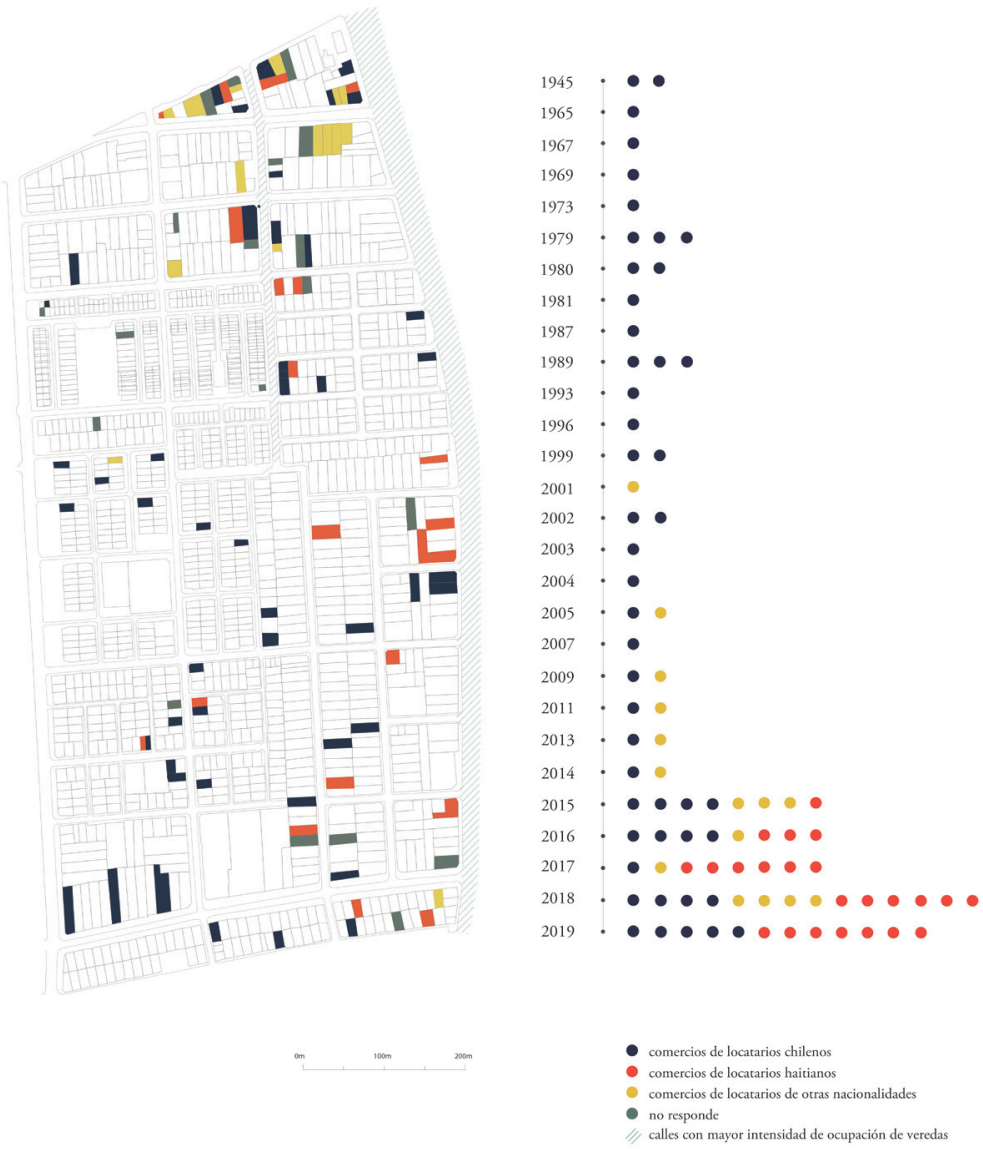

a) Distribución de locales comerciales, distin-

b) Línea de tiempo señalando ańo de apertura de guiendo entre locatarios chilenos, haitianos y comercio, distinguiendo entre locatarios chilenos, otros migrantes. haitianos y otros migrantes.

FUENTE: ELABORACIÓN PROPIA

En el Barrio v destacan principalmente los colectivos de origen haitiano y peruano, tanto por su número como por la visibilidad socioterritorial que manifiestan (Vargas et al., 2015). Una de las estrategias de inserción más característica que desarrolla la población caribeña es la relativa a los centros de belleza. Esto refleja la importancia del diseño del cuerpo en la cultura afrodescendiente, que los lleva a portar las costumbres de su territorio en el cuerpo (comunicación directa). Efectivamente, durante la última década ha sido posible observar en el barrio el surgimiento de numerosas peluquerías internacionales, que introducen nuevos estilos y costumbres al contexto chileno. 
Por su parte, entre las estrategias de inserción más usuales de la comunidad peruana en Chile están las prácticas relacionadas con la alimentación, que son visibles en el barrio a través de la introducción de comercios con productos alimentarios de su país de origen y la amplia oferta de restaurantes peruanos. Al decir de Imilan (2014), "el restaurante peruano es parte de un tipo de economía étnica, que deviene en una estrategia de inserción económica para los migrantes, cuyo acceso está mediado por la adscripción a una comunidad de origen, en este caso nacional” (p. 16).

El segundo tipo de espacios colectivos identificados en el Barrio v, los espacios emergentes, remite a apropiaciones del espacio público. En el caso de la población migrante, estas apropiaciones tienen, en su mayoría, fines comerciales, identificándose dos tipos de prácticas de carácter intermitente con gran visibilidad en el barrio. Una práctica de apropiación desarrollada principalmente por población migrante es la de los "coleros"; es decir, vendedores sin permiso oficial que se acoplan en los extremos de las ferias libres para aprovechar el flujo de personas, muchas veces duplicando la extensión de la feria. Otro tipo de espacios emergentes impulsados por migrantes está representado por veredas ocupadas por vendedores ambulantes con parrillas dedicadas a la preparación de comidas. Si bien estas ocupaciones tienen un fin económico, se transforman en puntos de encuentro y vitalidad para el barrio. También se identificaron apropiaciones de espacios públicos por parte de la población local, aunque la mayoría de estas presentan otras formas, como apropiaciones de la platabanda para estacionamiento o un jardín y, en menor medida, para fines comerciales.

Por último, el tercer tipo de espacios colectivos identificados fueron los espacios cotidianos, entendiendo por ellos los que albergan dinámicas diarias de sus habitantes. En el barrio, frecuentemente se utiliza el vano de la puerta y los peldaños de acceso a la casa como lugar de contemplación de la calle o como punto de sociabilización. De hecho, se observa que en las casas que no presentan peldańos de acceso, sus usuarios los reemplazan por sillas o cajas para sentarse. Se identifica que esta dinámica es desarrollada tanto por la población local del barrio como por la población migrante, por lo que se considera como un espacio con potencial integrador.

La distribución espacial de los espacios colectivos dejó ver una concentración de comercio peruano hacia el norte y de comercios haitianos hacia la zona oriente del barrio. Estas dos zonas albergan también una mayor intensidad de ocupación de veredas para fines comerciales y puntos de sociabilización (ver calles con achurado en la Figura 5a). Cabe destacar que es usual encontrar a grupos de haitianos reunidos en lugares colindantes a un comercio o una vivienda colectiva de connacionales. De hecho, algunos de ellos vienen desde otros sectores del barrio a estos puntos de sociabilización en la vía pública para reunirse con amigos connacionales, acompañados de música, y eludir de esta manera problemas con sus propios vecinos. Así, esta población caribeña recurre a la agrupación para demarcar fronteras y espacios de los cuales apropiarse. De acuerdo con Stefoni (2015), en el espacio urbano emerge la necesidad de un nosotros que, por un lado, reúne y, por otro, distingue del resto de la ciudad (p. 677). Esta apropiación de lugar y el conocimiento de las redes que en él se entretejen genera una sensación de seguridad.

Lo anterior nos permite leer fenómenos de segregación que, a la luz de Caggiano y Segura (2014), pueden entenderse por la intersección dinámica de distintas 
dimensiones; en este caso, de carácter social y cultural. Por un lado, la dimensión social, referida a la frecuencia de las interacciones entre los distintos grupos de una ciudad, se manifiesta en la población haitiana en un bajo nivel de cohesión social con otras nacionalidades, dificultada en gran parte por la barrera idiomática. Por otro, la dimensión cultural de la segregación, como aquellos aspectos sociales que definen la conformación de grupos, se refleja en demarcaciones por costumbres y raza. De hecho, en las entrevistas realizadas los sujetos se refieren a la existencia de discriminación para acceder a puestos de trabajo, frente a otras nacionalidades de migrantes.

La investigación a escala barrial muestra, así, un proceso de transformación urbana del barrio desencadenado por la espacialización de prácticas migrantes que han aportado a la reactivación de espacios subutilizados. La introducción de nuevos comercios, la resignificación de espacios urbanos tradicionales y la apropiación de veredas han llevado intensidad y diversidad a un barrio residencial que atraviesa un periodo de obsolescencia física. Véase al respecto Figura 6, donde se comparan dos fotos de una fachada en el Barrio v tomadas en 2015 y 2017. La imagen alude al proceso de densificación y activación del barrio.

Figura 6 | Comparación de la misma fachada del barrio, la primera del año 2015 (Google Earth) y la segunda capturada el 2017
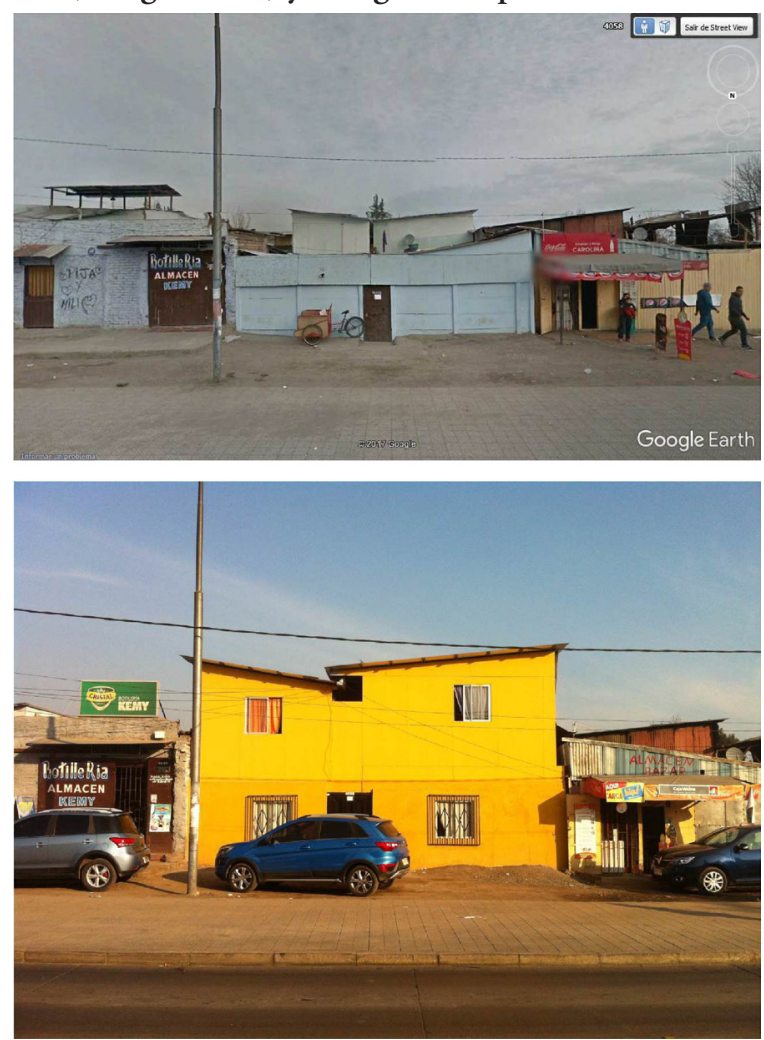

FUENTE: ELABORACIÓN PROPIA 


\section{Habitar temporal a escala doméstica}

La gran mayoría de los migrantes presenta soluciones habitacionales transitorias. Como lo indican Lafortune et al. (2016) en un estudio del Banco Interamericano de Desarrollo (BID), solo un 2\% de los inmigrantes tiene su vivienda propia en Chile, mientras que el resto presenta dinámicas habitacionales más flexibles o provisorias. Un $7 \%$ vive en casa compartida, un $8 \%$ vive en la casa de alguien como allegado, y un $80 \%$ arrienda (de los cuales el $38 \%$ arrienda una casa o departamento y el $42 \%$ arrienda una habitación).

De hecho, la situación más problemática de los migrantes se produce a nivel de vivienda. Las dificultades que enfrenta este grupo en términos económicos y legales para acceder al arriendo formal han provocado el surgimiento de un mercado de arriendo informal de habitaciones, que suele subdividir y ampliar precariamente viviendas sociales, casonas antiguas y galpones industriales. A estos se les ha llamado "nuevos conventillos", revisitando la tipología que surgió con las migraciones campo-ciudad de principios del siglo xix en la ciudad de Santiago (comunicación directa). Ello ha provocado que un 22,3\% de los hogares migrantes que residen en la Región Metropolitana habite en graves condiciones de hacinamiento (Encuesta de Caracterización Socioeconómica Nacional [CASEN], 2017).

Para profundizar en la escala doméstica del habitar migrante haitiano, se realizó el levantamiento de tres tipos de vivienda colectiva migrante presentes en el Barrio v, clasificadas según su origen: galpones industriales, casonas antiguas y vivienda social. Además, a modo de muestra control, se realizó el mismo ejercicio en una vivienda social habitada por una familia tradicional del barrio. La Figura 7 y la Figura 8 presentan las diferentes ampliaciones, indicándose en la línea de tiempo sus fechas y su carga de ocupación, representada en barras horizontales. En el caso de la muestra control, se consigna la cantidad de residentes de la vivienda en cada etapa; por su parte, en los casos migrantes, dada la variación de los integrantes de cada hogar -factor sumado a la barrera idiomática-, solo fue posible levantar la cantidad de hogares que habitan en ella y no cuántas personas.

La vivienda de muestra control era habitada desde 1957 por el mismo grupo familiar extendido, compuesto por 17 personas, con periodos de mayor y menor densidad habitacional (algunos nacen, mueren, se van y otros retornan). En cambio, en las viviendas colectivas habitadas predominantemente por migrantes haitianos, las distintas etapas de ampliaciones de las viviendas transcurren en espacios de tiempos más cortos (meses), mostrando un proceso de ocupación más dinámico. Además, cada hogar migrante arrienda una o dos habitaciones de la vivienda para todos sus integrantes, por lo que la mayoría de aquellas visitadas no presenta recintos exclusivos para dormir, concentrando en un mismo lugar las actividades de dormir, comer y sociabilizar. De esta manera, se difuminan los límites de lo íntimo y lo colectivo, constituyéndose las habitaciones como espacios multifuncionales.

Considerando que las migraciones contemporáneas se caracterizan por dinámicas como migración temporal, circular o de retorno, la gran mayoría de los hogares migrantes presenta variados cambios en su estructura a lo largo del tiempo, aumentando o disminuyendo los miembros del hogar. Mediante una serie de entrevistas realizadas en el barrio a 18 migrantes haitianos, se buscó ahondar en las trayectorias 
residenciales y las composiciones del hogar, detectándose dos situaciones muy habituales, a las cuales se les denominará “desconfiguración” y “reconfiguración” del grupo habitacional. Se optó por referirse al grupo como habitacional, ya que este puede o no tener vínculo familiar, así como compartir o no los gastos de alimentación (lo que tradicionalmente se entiende como hogar).

FIGURA 7 | Levantamiento de vivienda social habitada por familia chilena, comparada con una habitada por grupos habitacionales migrantes, y sus respectivas cargas de ocupación
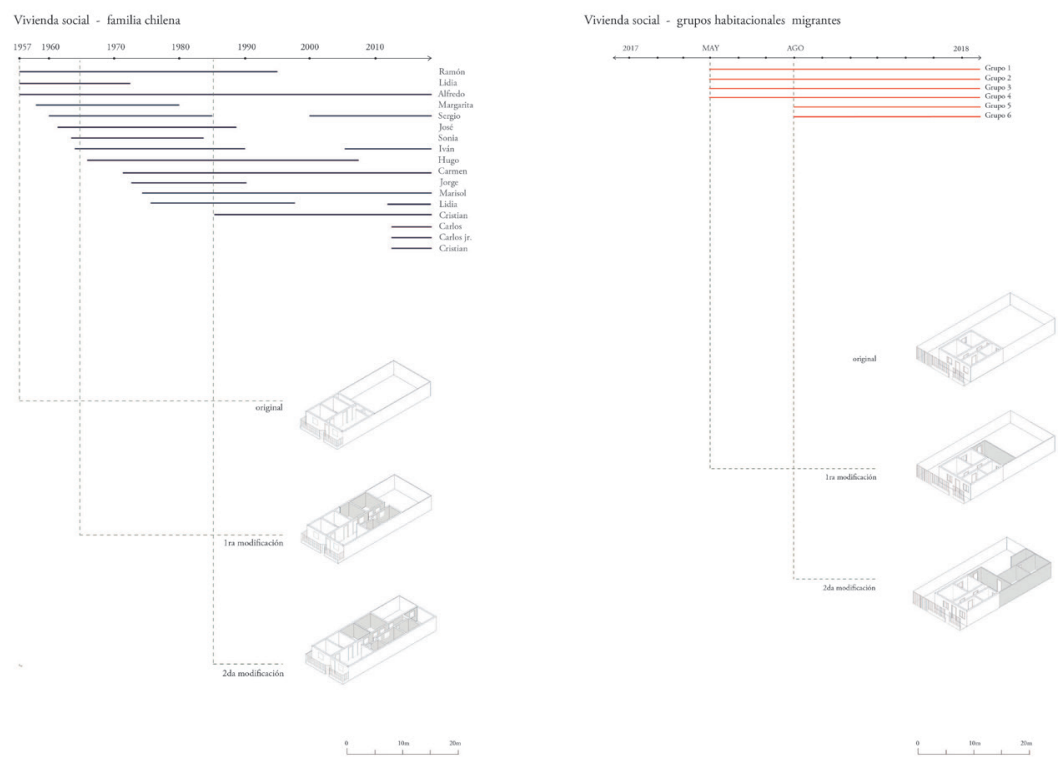

FUENTE: ELABORACIÓN PROPIA

La "desconfiguración” del grupo habitacional se refiere a la separación de los integrantes debido al proyecto migratorio, aunque sosteniendo un contacto frecuente a través de las redes sociales y, en muy pocos casos, viajes al país de origen. En el caso de los haitianos, la mayoría de los proyectos migratorios fueron iniciados por hombres, consolidándose así como el colectivo más masculinizado que recibe el país (INE, 2018), lo cual contrasta con la tendencia femenina de las actuales migraciones que recibe Chile. De los 18 haitianos y haitianas entrevistados, 6 indicaron tener al menos un hijo viviendo en Haití. Concordante a esto, el estudio realizado por el BID (Lafortune et al., 2016) señala que un $52 \%$ de las mujeres y $60 \%$ de los hombres migrantes latinoamericanos con hijos tiene al menos un hijo viviendo en el país de origen. 
FIGURA 8 | Levantamiento de galpón industrial y casona antigua, ambas habitadas por grupos habitacionales migrantes, y sus respectivas cargas de ocupación
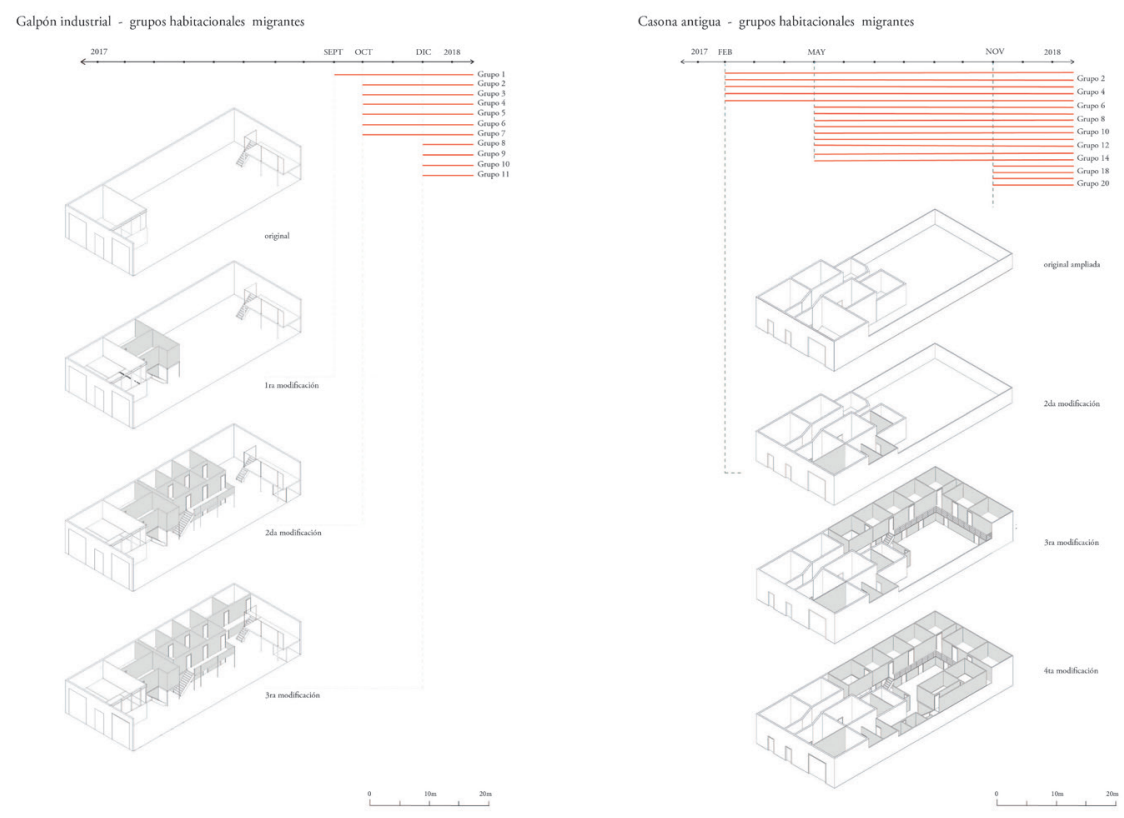

FUENTE: ELABORACIÓN PROPIA

La "reconfiguración" del grupo habitacional se refiere a una relación de convivencia de personas unidas por lazos de parentesco, amistad o adscripción a una comunidad de origen; en palabras de Mercado (1993, citado en Tapia et al., 2012), "con el fin de maximizar recursos económicos, habitacionales y sociales". Esta situación es especialmente frecuente en los primeros ańos del proyecto migratorio; de hecho, el Censo 2017 muestra que mientras más reciente es la migración, mayor es la presencia de "otro pariente" o "no pariente" en el hogar (INE, 2017). De los migrantes que llegaron a Chile antes del 2010, un 2,7\% presenta una relación de "no pariente" en el hogar, cifra que asciende a 22,8\% entre los que ingresaron al país el año $2017^{1}$ (INE, 2018). Según el Censo 2017, Haití es el colectivo con mayor cantidad de "no parientes", situación que también se vio fuertemente reflejada en las entrevistas, donde 7 de los 18 entrevistados haitianos dice haber vivido con algún "no pariente". Esta situación demuestra cómo la migración contemporánea trasciende los vínculos familiares, operando muchas veces por medio de redes de amistad o adscripción a la misma comunidad de origen.

Las migraciones a escala doméstica revelan un estilo de vida de constantes variaciones tanto en términos de composición del grupo habitacional como de transformaciones espaciales y materiales (Figura 9). Esto manifiesta el dinamismo intrínseco

1 El año 2017 solo considera hasta el 19 de abril, día de la aplicación del censo. 
a la condición migrante y, a la vez, caracteriza los modos de habitar contemporáneos, lo que levanta la inquietud por responder con soluciones habitacionales a estos flujos y nuevas composiciones del hogar.

FIGURA 9 | Espacio multifuncional divido por cortinas
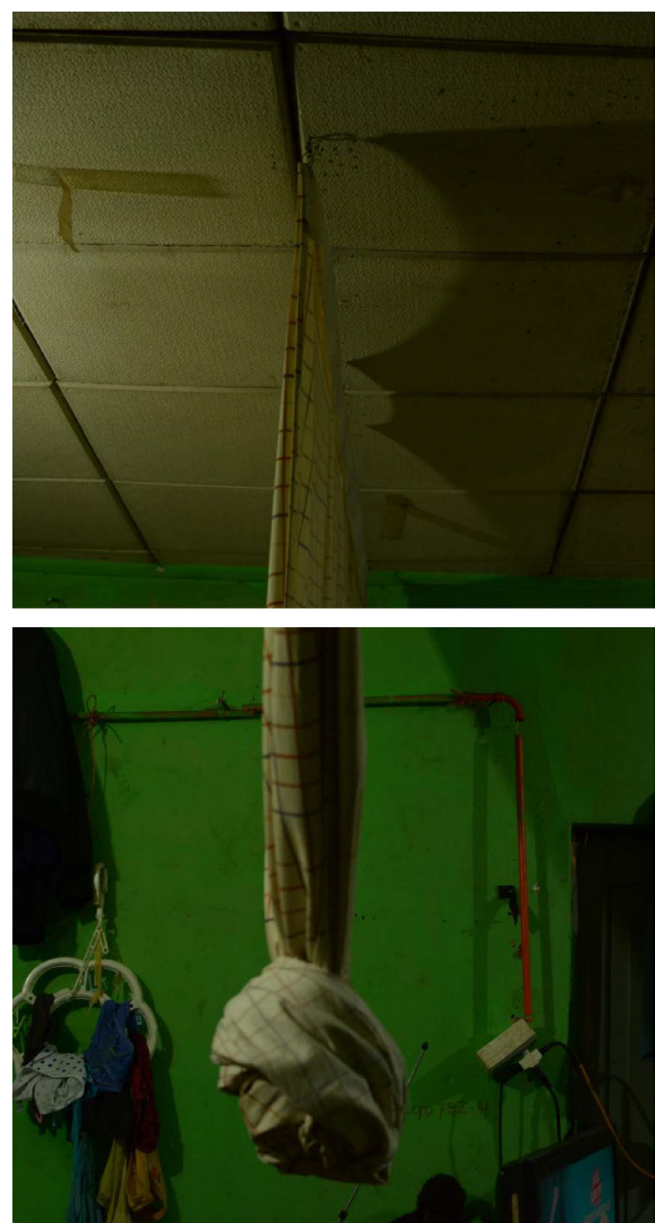

FUENTE: ARCHIVO PERSONAL

\section{Conclusiones}

El presente artículo, enfocado en las migraciones provenientes de América Latina y el Caribe, entrega un análisis descriptivo del mayor flujo migratorio que ha recibido el país. Este es relevante cuantitativamente, por su aumento explosivo, pero también en términos cualitativos, ya que en la medida en que se han diversificado las nacionalidades que llegan al país, han cambiado los patrones socioculturales e idiomáticos (DEM, 2016). 
El estudio del fenómeno migratorio haitiano en el Gran Santiago desde una aproximación multiescalar permite ver una interrelación entre las tres escalas abordadas y da cuenta de cómo las redes interpersonales organizan espacialmente las migraciones y modelan los espacios en todos sus niveles. Al decir de Imilan et al. (2014), las redes, además de cumplir roles funcionales, estructuran la vida social en su conjunto; integran y reúnen en torno al sentido de pertenencia; y, al mismo tiempo, diferencian y refuerzan la especificidad cultural frente a la otredad. De esta manera, las relaciones estructuradas por las redes no quedan anidadas en escalas específicas, sino que todos los espacios en que se dan se ven coproducidos por las mismas relaciones sociales (Brenner, 2011).

A nivel de ciudad, las redes agrupan a los migrantes, expresándose espacialmente en tendencias de localización y concentraciones desproporcionadas en determinadas zonas de acuerdo con el origen. La mayoría de los colectivos estudiados muestra una tendencia común de concentración en las comunas centrales de Santiago. En la comunidad haitiana, en cambio, se observa una mayor dispersión en la ciudad, alcanzando sus mayores concentraciones en comunas distantes entre sí, varias de las cuales recibieron flujos tempranos de este colectivo. Así, los migrantes haitianos llegan a prácticamente a todas las comunas de la Región Metropolitana e incluso a las zonas periurbanas, con la sola excepción del cono de alta renta; sin embargo, esta distribución no puede describirse como un patrón disperso, ya que estos migrantes tienden a agruparse con connacionales. Tal tendencia no se manifiesta solamente a nivel de ciudad, sino también a escala del barrio, en ciertas calles o zonas, y en la vivienda, donde las personas se agrupan con otros connacionales. Esta es una manifestación de las redes funcionando multiescalarmente.

Por otra parte, el análisis del barrio muestra cómo las redes, en la medida en que constituyen flujos sociales y culturales, articulan las prácticas colectivas adquiriendo fuerte expresión en el espacio urbano. Tales prácticas se han manifestado en la introducción de costumbres específicas a las culturas de origen, apropiaciones de vereda y demarcaciones de fronteras ligadas a las zonas de comercios y viviendas migrantes. Es interesante destacar que la mayor parte de los espacios compartidos y emergentes impulsados por la comunidad haitiana en el barrio se asocian a estrategias de inserción económica, mientras los espacios cotidianos, que tienen lugar en los vanos de acceso a la vivienda, tienen un tinte más social. Cada uno de ellos alimenta y agrega vitalidad a barrios que, a su llegada, ostentaban señales de obsolescencia. Es decir, si bien parte importante de las prácticas migrantes en el espacio público se encuentran asociadas a fines comerciales, su principal impacto ha sido la activación de espacios subutilizados y la resignificación de espacios urbanos tradicionales. Esto concuerda con lo descrito por Saskia Sassen, quien ha destacado procesos de revitalización de barrios producto del dinamismo económico migrante (Segura \& Abde, 2014).

La escala doméstica da cuenta de cómo los patrones migratorios contemporáneos han dado origen a nuevas configuraciones del grupo habitacional, las que muchas veces trascienden los lazos familiares, desarrollándose a partir de redes entre integrantes de una misma comunidad de origen. Esto ha dado pie a estilos de vida donde las variaciones del grupo habitacional y las transformaciones materiales de sus espacios domésticos se han vuelto una realidad cotidiana. 
La observación de la variable temporal en el análisis del espacio urbano contemporáneo revela el dinamismo intrínseco de la condición migrante, donde los estilos de vida se caracterizan por la movilidad frente al asentamiento y lo cambiante frente a lo definitivo. Estos aspectos, a su vez, cristalizan rasgos propios de la sociedad contemporánea y tensionan la concepción estática de las ciudades: lo que antes se vivía en ańos, pareciera que ahora sucede en meses. De acuerdo a Mehrotra (2015), este dinamismo exige concepciones urbanísticas que creen entornos lo suficientemente robustos y versátiles para que la condición cinética de la ciudad se desarrolle. Así, se sostiene que nunca antes había sido tan importante avanzar en el desarrollo de estrategias urbanas que promuevan la accesibilidad, movilidad residencial y flexibilidad de los espacios privados y colectivos, permitiendo acoger distintas identidades culturales, rangos temporales y variaciones de los grupos habitacionales.

\section{Agradecimientos}

Investigación dedicada a todas y todos aquellos cuyo norte es el sur.

Las autoras quieren agradecer especialmente a la Estación Intercultural de la comuna de Estación Central, Puentes uc y al Servicio Jesuita a Migrantes, por la gran labor que realizan y el apoyo recibido en esta investigación. También a Walter Imilan, que, como académico experto en temas de movilidad y migraciones, entregó valiosa información; a Pablo Salvador Banda, arquitecto que aportó en la programación visual de datos para la elaboración de las planimetrías a nivel de ciudad; y a Sebastián Salas, fundador de Linchpin, cuyo pensamiento crítico fue de gran relevancia. Asimismo, se agradece el apoyo para la investigación proporcionado por el CEDEUS, ANID/FONDAP I 5 I IOO20 y el proyecto FONDECYT I I 90850 "Densidad, Diversidad y Accesibilidad: claves para un desarrollo urbano sustentable”.

\section{Referencias bibliográficas}

Bovenkerk, F. (1974). The sociology of return migration: A bibliographic essay. Martinus Nijhoff. Brenner, N. (2011). The urban question and the scale question. Some conceptual clarifications. En A. Çağlar \& N. G. Schiller (Eds.), Locating migration. Rescaling cities and migrants (pp. 23-40). Cornell University Press. https://doi.org/10.7591/9780801460340-003

Caggiano, S. \& Segura, R. (2014). Migración, fronteras y desplazamientos en la ciudad. Dinámicas de la alteridad urbana en Buenos Aires. Revista de Estudios Sociales, (48), 29-42. http://dx.doi.org/10.7440/res48.2014.03

Çağlar, A. \& Schiller, N. G. (2011). 1. Introduction: Migrants and cities. En A. Çağlar \& N. G. Schiller (Eds.), Locating migration. Rescaling cities and migrants (pp.1-20). Cornell University Press. https://doi.org/10.7591/9780801460340-002

Çağlar, A. \& Schiller, N. G. (2018). 1. Introduction: Multiscalar city-making and emplacement: processes, concepts, and methods. En A. Çağlar \& N. G. Schiller (Eds.), Migrants and city-making: Dispossession, displacement, and urban regeneration (pp. 1-31). Duke University Press. 
Chiappini, M. C., Scheerlinck, K. \& Schoonjans, Y. (2016). Configuración espacial de espacios colectivos dentro de dinámicas formativas. La relación entre la infraestructura y la transformación urbana en el caso de Plaza de las Glorias Catalanas. Arquisur Revista, 6(9), 56-75. https://doi.org/10.14409/ar.v0i9.5797

Departamento de Extranjería y Migración (DEM), Chile. Estadísticas migratorias. https://www. extranjeria.gob.cl/estadisticas-migratorias/

Departamento de Extranjería y Migración (DEM), Chile. (2016). Migración en Chile 20052014. Ministerio del Interior y Seguridad, Chile.

Encuesta de Caracterización Socioeconómica Nacional (CASEn). (2017). Véase Ministerio de Desarrollo Social y Familia, Observatorio Social.

Imilan, W. (2014). Restaurantes peruanos en Santiago de Chile: Construcción de un paisaje de la migración. Revista de Estudios Sociales, (48), 15-28. https://doi.org/10.7440/ res48.2014.02

Imilan, W., Garcés, A. \& Margarit, D. (Eds.). (2014). Poblaciones en movimiento. Etnificación de la ciudad, redes e integración. Ediciones Universidad Alberto Hurtado.

Instituto Nacional de Estadísticas (INE), Chile. (2017). Censo 2017. Resultados. Región Metropolitana de Santiago. http://resultados.censo2017.cl/Region?R=R13

Instituto Nacional de Estadísticas (INE), Chile. (2018). Características de la inmigración internacional en Chile, Censo 2017. https://www.censo2017.cl/descargas/ inmigracion/181123-documento-migracion.pdf

Lafortune, J., Tessada, J., Rouse, R. \& Keller, L. (2016). Migrantes latinoamericanos en Chile: un panorama de su integración social, económica y financiera. Banco Interamericano de Desarrollo (віD).

Lefebvre, H. (1991). The production of space. Trad. por D. Nicholson-Smith. Blackwell. https:// monoskop.org/images/7/75/Lefebvre_Henri_The_Production_of_Space.pdf

Marcos, M. \& Mera, G. (2018). Migración, vivienda y desigualdades urbanas: condiciones socio-habitacionales de los migrantes regionales en Buenos Aires. Revista INVI, 33(92), 53-86. https://doi.org/10.4067/s0718-83582018000100053

Massey, D., Arango, J., Hugo, G., Kouaouci, A., Pellegrino, A. \& Taylor, J. (1993). Theories of international migration: A review and appraisal. Population and Development Review, 19(3), 431-466. https://doi.org/10.2307/2938462

Mehrotra, R. (2015). La ciudad cinética como generadora de práctica. Ediciones ARQ.

Mehrotra, R. (2016). Trabajar en Bombay: la ciudad como un generador de práctica. Dearq, 18, 88-95. https://revistas.uniandes.edu.co/doi/pdf/10.18389/dearq18.2016.07a

Mercado, O. (1993). El allegamiento: operacionalización del concepto y características relevantes. En Ministerio de Vivienda y Urbanismo, Chile, División Técnica de Estudio y Fomento Habitacional, Seminario Allegados: caracterización y propuesta para una politica de vivienda (pp. 31-38). Santiago, 28 y 29 de octubre, 1993.

Organización Internacional para las Migraciones (oIM). (2009). Migración laboral, temporal y circular: experiencias, retos y oportunidades. oIm / Unión Europea. https:// repositoryoim.org/bitstream/handle/20.500.11788/238/COL-OIM\%200321\%20 N2-1.pdf?sequence=1 \&isAllowed $=y$

Pries, L. (2005). Configurations of geographic and societal spaces: A sociological proposal between 'methodological nationalism' and the 'spaces of flows'. Global Networks, 5(2), 167-190. https://doi.org/10.1111/j.1471-0374.2005.00113.x 
Schiller N. G., Basch, L. \& Blanc-Szanton, C. (1992). Towards a definition of transnationalism: Introductory remarks and research questions. Annals of the New York Academy of Sciences, 645(1), ix-xiv. https://doi.org/10.1111/j.1749-6632.1992.tb33482.x

Segura, D. M. \& Abde, K. B. (2014). Barrios y población inmigrantes: El caso de la comuna de Santiago. Revista INVI, 29(81), 19-77. http://dx.doi.org/10.4067/S071883582014000200002

Solá Morales, M. (1992). Espacios públicos y espacios colectivos. La Vanguardia, 12/05/1992, p. 4. http://hemeroteca-paginas.lavanguardia.com/LVE08/HEM/1992/05/12/SU11 9920512-004.pdf

Stefoni, C. (2004). Inmigrantes transnacionales: la formación de comunidades y la transformación en ciudadanos. En U. D. Berg \& K. Paerregaard (Eds.), El Quinto Suyo: Transnacionalidad y formaciones diaspóricas en la migración peruana (Cap. 9). Facultad Latinoamericana de Ciencias Sociales, Flacso Chile. Artículo en http:// biblioteca.clacso.edu.ar/ar/libros/chile/flacso/artstef.pdf

Stefoni, C. (2015). Reconfiguraciones identitarias a partir de habitar el espacio público: el caso de los migrantes esquineros en la ciudad de Santiago, Chile. Chungara. Revista de Antropología Chilena, 47(4), 669-678. https://doi.org/10.4067/s071773562015005000035

Tapia, R., Araos, C. \& Salinas, C. (2012). Condominios familiares. Una alternativa de solución residencial para familias allegadas en lotes tipo 9x18. En Centro de Políticas Públicas uc, Propuestas para Chile. Concurso Politicas Públicas 2012. Pontificia Universidad Católica de Chile. https://politicaspublicas.uc.cl/wp-content/uploads/2015/02/ propuestas-para-chile-2012-capitulo-iv.pdf

Urbina, A. (2016). Densificación como construcción de resiliencia. Barrio de Lourdes, Quinta Normal (Tesis de maestría inédita). Pontificia Universidad Católica de Chile, Santiago, Chile.

Vargas, I., Leguía, M. \& Villarroel, S. (2015). Informe técnico. Programa piloto de mediadores interculturales PIMSI. Municipalidad de Estación Central. 